\title{
Preference Models Used in Multiple Criteria Decision Making: Foundations and Assessment
}

\author{
Denis Bouyssou \\ CNRS-LAMSADE, Université Paris Dauphine, \\ F-75775 Paris Cedex 16, France \\ bouyssou@lamsade.dauphine.fr
}

\begin{abstract}
This purpose of this talk is to offer a nontechnical introduction to the main preference models used in multiple criteria decision making. The emphasis is on the, central, additive value function model. We outline its axiomatic foundations and present various possible assessment techniques to implement it. Some extensions of this model, e.g., nonadditive models or models tolerating intransitive preferences are then briefly reviewed.
\end{abstract}

